\title{
Cálculo de frenagem em um software didático
}

\author{
Anibal Osório de Lima Junior ${ }^{1}$, Tatiana Annoni Pazeto ${ }^{2}$ \\ ${ }^{1}$ Curso de Bacharelado em Engenharia Mecânica - Universidade Federal de Rondonópolis \\ (UFR) \\ ${ }^{2}$ Curso de Bacharelado em Sistemas de Informação - Universidade Federal de \\ Rondonópolis (UFR) \\ 78735-901 - Rondonópolis - MT-Brasil aniballima97@gmail.com, \\ tpazeto@gmail.com.
}

\begin{abstract}
Due the difficulties in finding programs that present data on brake systems and the limitation in the application of the physics discipline in high school, a software with this purpose was developed. For this, bibliographical researches that address the themes were analyzed, as well as websites of vehicle assemblies. The results of this study culminated in a didactic software that takes up the contents of the physics discipline, in addition to conducting calculations and defining which variable act during the braking process.
\end{abstract}

Resumo. Visto as inúmeras dificuldades em encontrar programas que apresentam dados sobre os sistemas de freios e uma limitação na aplicação da disciplina de física no ensino médio, desenvolveu-se um software com esse intuito. Para isso, pesquisas bibliográficas que abordam o tema foram analisadas, bem como sites das montadoras de veículos. Os resultados deste estudo culminaram em um software didático que retoma os conteúdos da disciplina de fisica, além de realizar cálculos de forças e variáveis que atuam durante o processo de uma frenagem.

\section{Introdução}

Ao longo da evolução histórica houve diversos avanços como a criação das rodas, motores e veículos, visando facilitar a locomoção de pessoas. Destaca-se o transporte de cargas, que inicialmente era executado por seres humanos. Tempos mais tarde, com a domesticação dos animais, o transporte passou a ser através da tração animal, usando sistemas de rodas que propiciaram locomover cargas cada vez maiores. Entretanto, com a utilização das rodas e o aumento das cargas notou-se a necessidade de algum mecanismo para retirar ou reduzir o movimento dos corpos [Maluf et al, 2007].

A partir dessas premissas surgiu a ideia para a criação de um sistema de freios. Seguindo esse histórico e com o intuito de calcular as forças atuantes na frenagem, bem como aumentar a interação dos alunos na disciplina de física, foi produzido um software para a determinação das forças atuantes ao longo desse processo.

Para isso, pesquisas bibliográficas sobre o sistema de freio foram realizadas para compreender o funcionamento e como seria possível calcular um processo de frenagem através dos conteúdos da disciplina de física. Nesse processo ocorreram diversas dificuldades devido a abrangência do tema, dificultando a sua delimitação. Após o levantamento bibliográfico e o entendimento do funcionamento do cálculo de frenagem, a parte prática foi realizada. A mesma consiste em desenvolver um software didático, na linguagem C, para aumentar o interesse na disciplina de física. Essa etapa ocorreu durante o primeiro semestre de Engenharia Mecânica, com o aprendizado do conteúdo de algoritmo.

$\mathrm{O}$ artigo está estruturado em seis seções. Na Seção 2 apresenta-se o embasamento matemático necessário para a criação do software. A Seção 3 versa sobre trabalhos correlatos que serviram de base para o desenvolvimento do projeto. A interface, funcionamento do 
programa e uma simulação são descritos na Seção 4. As conclusões constam na sessão 5. Por fim, as referências são apresentadas.

\section{Fundamentação teórica}

O princípio para a realização do cálculo de frenagem é encontrado na disciplina de física do Ensino Médio, bem como no conteúdo programático de cursos de graduação como de Engenharia Mecânica e Engenharia Automotiva.

Nesta seção do artigo serão apresentados os fundamentos teóricos utilizados para o desenvolvimento de um software didático para o cálculo da força de frenagem, do tempo de frenagem, da distância total para o veículo ficar estático e a eficiência do freio.

A segunda lei de Newton, também conhecida como princípio fundamental da dinâmica, permite definir a força para romper a inércia de um corpo [Newton, 2002]. A mesma é apresentada na Equação 1.

$$
\begin{gathered}
F=m * a \\
F=\text { Força de frenagem } \\
m=\text { Massa do corpo } \\
a=\text { Desaceleração }
\end{gathered}
$$

Fonte: Newton (2002).

\section{Equação 1: Equação da segunda lei de Newton.}

Afim de realizar o cálculo da aceleração para aplicar na Equação 1, foi construída uma relação em cadeia, na qual define-se variáveis, como por exemplo, a distância percorrida durante o processo de frenagem (Equação 2), que aplicada na equação de Torricelli (Equação 3) resulta na aceleração/desaceleração de um corpo.

Durante o cálculo da distância (Equação 2) são necessários dados como a velocidade e o coeficiente de atrito. Para obter os valores para o coeficiente de atrito, a serem utilizados nos cálculos do software, foram inseridas informações da Tabela 1 [Rosa; Leal; Nicolazzi, 2008].

Tabela1: Coeficientes de atrito.

\begin{tabular}{|c|c|}
\hline Tipo de Pista & $\mu$ \\
\hline Asfalto & 0.60 a 0.95 \\
\hline Pedra britada & 0.50 a 0.65 \\
\hline Terra seca & 0.50 a 0.60 \\
\hline Terra úmida & 0.50 a 0.60 \\
\hline Areia & 0.20 a 0.30 \\
\hline Neve & 0.30 a 0.25 \\
\hline
\end{tabular}

Fonte: Rosa; Leal; Nicolazzi (2008).

Para obter a distância total deve-se considerar o tempo de reação do condutor até o acionamento do freio que, segundo Rodrigues (2002), é de aproximadamente 0,3 a 0,7 segundos. Adequando a Equação 2 ao tempo de reação do motorista, é calculada a distância percorrida ao longo do tempo de reação e a partir da velocidade. Posteriormente, adicionase a distância de reação na Equação 2 para o cálculo da distância total visto que essa possui seu resultado já em metros.

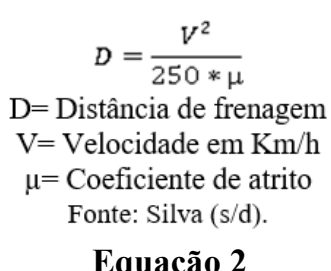

Equação 2

$$
\begin{aligned}
& V^{2}=V_{0}^{2}+2 * a * \Delta s \\
& V=\text { Velocidade final } \\
& V_{\circ}=\text { Velocidade inicial } \\
& \alpha=\text { Desaceleração } \\
& \Delta s=\text { Variação de espaço } \\
& \text { Fonte: Sales, Maia (2011, p.35) } \\
& \text { Equação } 3
\end{aligned}
$$

Equação 2: Cálculo da distância; Equação 3: Equação de Torricelli.

Considerando o valor obtido na variação total do espaço percorrido pelo veículo 
durante a frenagem, é possível calcular a aceleração/desaceleração através da Equação 3, posto que o cálculo possibilita encontrar essa variável através da modificação do posicionamento do corpo, da velocidade inicial e final.

Realizado o cálculo da aceleração e também a variação de espaço, é possível conhecer o tempo estimado para a fricção total do veículo. Afinal, o tempo pode ser determinado através da aplicação dos dados na fórmula de Aceleração Escalar Média (Equação 4), na qual a aceleração é a taxa de variação de velocidade em um tempo delimitado.

$$
\begin{gathered}
a=\frac{\Delta s}{\Delta t} \\
\alpha=\text { Aceleração/Desaceleração } \\
\Delta s=\text { Variação de espaço } \\
\Delta \mathrm{t}=\text { Variação de tempo } \\
\text { Fonte: Silva }(\mathrm{s} / \mathrm{d}) . \\
\text { Equação } 4
\end{gathered}
$$

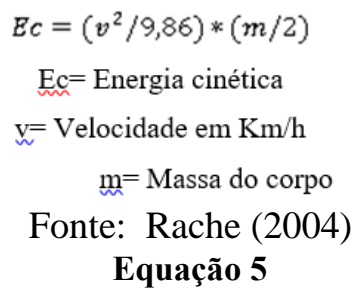

Equações 4: Aceleração escalar média; Equação 5: Energia Cinética;

\section{Equação 6: Eficiência do freio}

Para obter o tempo de frenagem utiliza-se a Equação 4, que através da variação do espaço e da aceleração, e considerando a existência de 0,7 segundos de tempo de reação previsto por Rodrigues (2002), é possível obter o tempo total para a desaceleração. Para a determinação do total de energia cinética necessária durante o processo de frenagem é utilizada a Equação 5. Posteriormente a energia cinética será transformada em energia térmica durante o processo de fricção (Frenagem).

Ademais ocorrerá o cálculo da eficiência do freio (Equação 6) que pode ser obtido através do estudo sistemático do funcionamento do sistema de frenagem em uma pista de rolamento, na qual se averigua o estado do freio através de testes que observa a velocidade e o tempo de frenagem. Dessa forma obtém-se a porcentagem da eficiência do freio que, segundo a norma da Organização Internacional para Padronização (ISO 3450, 2011), deve ser acima de $28 \%$, afim de considerá-lo um freio eficiente.

\section{Trabalhos correlatos}

Bornhold (2012) relata sobre o desenvolvimento de um sistema de freios e os cálculos necessários, descrevendo detalhadamente o funcionamento e a importância de todos os mecanismos de um sistema de freio, os tipos e a aplicação dos cálculos para o desenvolvimento de um novo sistema de freios. Além disso, apresenta relatos sobre experiências com o desenvolvimento de sistemas de freios no projeto de extensão Baja SAE Brasil. Analisando esse trabalho foi possível extrair as ideias bases para a utilização das fórmulas no cálculo de frenagem de um veículo.

Santos (2014), descreve sobre a dinâmica veicular durante a frenagem, ressaltando tópicos a respeito da transferência longitudinal de carga, dos componentes do sistema de freios e os tipos de sistema de freios. Para Santos (2014), o cálculo para desaceleração veicular (Equação 3) é obtido através da velocidade inicial, visto que ao longo da frenagem pode ser considerado como desaceleração constante. Dessa forma é possível calcular a distância de frenagem (Equação 2). Considerando esses dados foi possível estabelecer um método para descobrir as variáveis de aceleração/desaceleração e a distância de frenagem.

O SIMRET 4000 (2021) é um equipamento desenvolvido pela empresa Turnkey Instruments Ltd. Ele é comumente usado para a realização de teste de eficiência nos sistemas de freios. Através de informações disponibilizadas no site da empresa, foi possível perceber que para a obtenção dos resultados da eficiência de um freio são realizados cálculos 
correlacionando a velocidade e a distância durante a frenagem de um veículo.

\section{Desenvolvimento do projeto}

A partir dos dados levantados nos trabalhos de Bornhold (2012), Santos (2014) e ao funcionamento do equipamento SIMRET 4000 (2021) foi desenvolvido um software, na linguagem $\mathrm{C}$, para a determinação de alguns dados ao longo de um processo de frenagem.

Bornhold (2012) desenvolveu os cálculos para a determinação dos esforços para um veículo específico, com determinados valores de massa. O software desenvolvido irá funcionar de maneira semelhante, utilizando os dados de massa inseridos na programação, podendo simular o equacionamento das forças de frenagem em condições diversas.

Analisando o trabalho de Santos (2014) foi possível pesquisar métodos para encontrar as variáveis de desaceleração e distância de frenagem que são obtidas através das Equações 2 e 3, além de complementar a ideia inicial presente na Equação 1.

Analogamente ao SIMRET 4000 (2021), o software funciona realizando relações semelhantes as desenvolvidas pelo equipamento, pois ambos dependem das variáveis distância percorrida e velocidade. Através dessas variáveis o equipamento é capaz de calcular a eficiência do freio em estudo. Seguindo esse mesmo princípio, o software também é capaz de realizar cálculos semelhantes que convergem na eficiência do freio previsto na Equação 6. No entanto, não foi possível determinar a diferença percentual entre o software e o equipamento, visto que em SIMRET 4000 (2021) não há possibilidade de realizar testes.

Visando complementar e possibilitar a execução dessas ideias, foram incluídas as demais equações. Como exemplo, a Equação 4 viabiliza calcular a variação de tempo necessário para a frenagem e a Equação 5 permite obter a energia cinética necessária para frear o veículo.

Com o propósito de realizar os cálculos através de um software, desenvolvido na linguagem C, utilizou-se estruturas de repetições, de controle e seleção, funções, vetores e bibliotecas especiais. Essas bibliotecas possibilitam alterar as cores da interface do programa, a inclusão de sinais sonoros, retorno ao menu e comando para a limpeza da tela.

Para facilitar a simulação realizada pelo usuário foram inseridos modelos de carros com suas respectivas massas, as quais foram obtidas a partir dos sites das montadoras. Os coeficientes de atritos já estão armazenados no programa, bastando ao usuário selecionar a opção desejada. Os dados referentes as massas dos veículos foram obtidos nos sites das montadoras Chevrolet (2021), Fiat (2021), Ford (2021), Honda (2021), Hyundai (2021) e Toyota (2021). Salienta-se que nas referências constam apenas de um modelo de cada veículo de cada montadora. Mas para todos os veículos disponíveis no programa o mesmo procedimento foi usado. Já os coeficientes de atrito foram extraídos da Tabela 1.

Simulando a execução do software, foi utilizado o usuário "Mateus", a montadora "Chevrolet", a categoria "Picapes", o veículo "S10 cabine dupla" considerando-o carregado com sua "carga máxima" e o terreno "Terra seca". Essa simulação pode ser visualizada nas

Figuras 1 e 2.

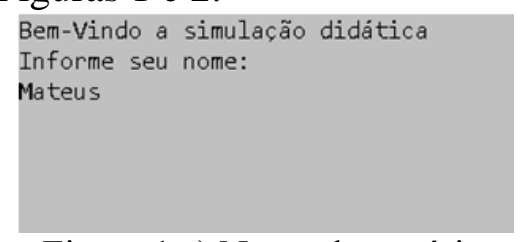

Figura 1 a) Nome do usuário

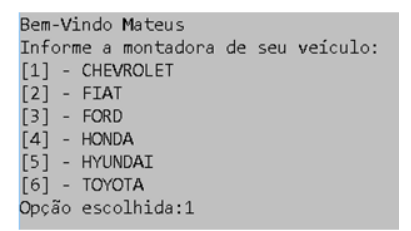

Figura 1 b) Seleção da montadora

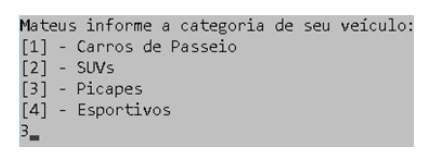

Figura 1 c) Seleção da categoria do veículo

\section{Figura 1: Seleções de: a) Nome do usuário; b) Montadora; c) Categoria do veículo}

A Figura 1 apresenta prints da tela das três primeiras interfaces do programa, sendo que, como pode ser observado na Figura 1 a) o usuário insere o seu nome, como "Mateus". 
Em seguida, na Figura 1 b), deve ser informada a montadora desejada para a execução da simulação, sendo selecionada a opção número 1 que se refere a Chevrolet. Já a Figura 1 c) ilustra a categoria desejada pelo usuário, sendo selecionada a opção 3, ou seja, Picapes.

$\mathrm{Na}$ Figura 2 são apresentadas as demais seleções para posterior execução do programa.

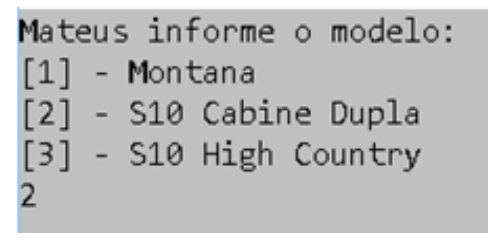

Figura 2 a) Seleção do modelo
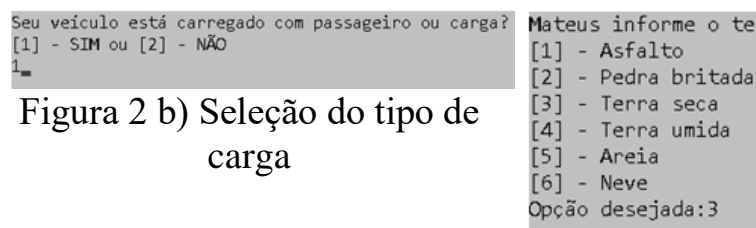

Figura 2 c) Seleção do terreno

\section{Figura 2: Seleções de: a) Modelo; b) Tipo de carga; c) Tipo do terreno}

Na Figura 2 são apresentados os recortes de telas para a seleção das opções para a execução do programa. Na Figura 2 a) o usuário deve escolher o modelo, sendo do tipo S10 Cabine Dupla. Posteriormente, na Figura 2 b), deve-se informar se o carro está carregado com passageiro ou carga. Por fim, na Figura 2 c), o usuário deve selecionar o coeficiente de atrito para a realização da simulação, sendo que a opção escolhida no exemplo é Terra seca.

Para dar segmento, o usuário ainda precisa informar a velocidade e o cálculo que deseja que seja executado. Posteriormente, os resultados são informados. Esses processos são apresentados na Figura 3.

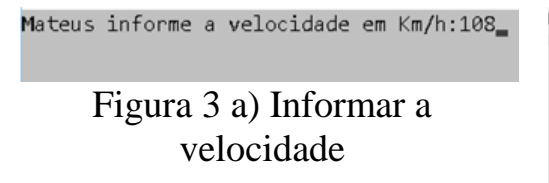

velocidade

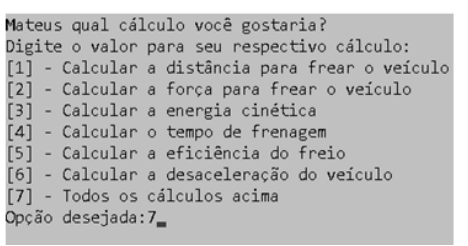

Figura 3 b) Informar o cálculo a ser realizado

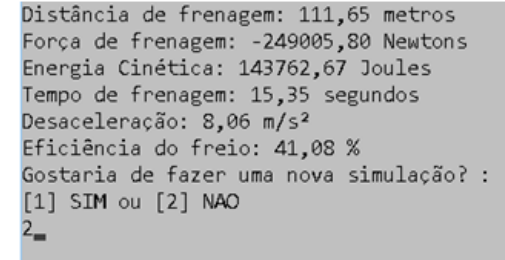

Figura 3 c) Resultados do cálculo selecionado

Figura 3: Seleções e resultados: a) Velocidade; b) Cálculo que deseja; c) Resultados do cálculo selecionado.

Conforme a Figura 3 a) é informado uma velocidade de $108 \mathrm{~km} / \mathrm{h}$. O tipo de cálculo que deve ser realizado é ilustrado na Figura 3 b). Em seguida, todos os cálculos relativos a seleção escolhida na Figura 3 b) são realizados e apresentados (Figura 3 c)).

A fim de que o software obtenha todos os valores, inicialmente calcula-se a distância percorrida, através da Equação 2, que será igual a 111,65 metros. Aplicando esse resultado na equação de Torricelli, Equação 3, obtém-se uma desaceleração igual a $-8,06 \mathrm{~m} / \mathrm{s}^{2}$. Antes de realizar o cálculo da força é preciso multiplicar a massa armazenada pelo programa pela aceleração da gravidade de $9,80665 \mathrm{~m} / \mathrm{s}^{2}$, que resulta em 30890,94 Newtons. Aplicando os resultados obtidos na Equação 1, chega-se ao valor de - 249005.80 Newtons que será a força necessária para frear o veículo.

Com os resultados referentes a variação de espaço e a aceleração é possível determinar o tempo total para a fricção, que será de 15,35 segundos (Equação 4). Utilizandose das informações fornecidas pelo usuário do software, calcula-se o valor da energia cinética (Equação 5) que será igual a 143762,67 Joules e a eficiência do freio (Equação 6) que será igual a $41,08 \%$. Todas essas informações podem ser observadas nos recortes de tela do programa apresentados na Figura $3 \mathrm{c}$ ). 
Vale salientar que para o desenvolvimento do programa e comparação com os resultados obtidos no mesmo, os cálculos foram resolvidos analiticamente. Além disso, o programa foi testado por alguns engenheiros mecânicos.

\section{Conclusão}

O presente trabalho objetivou desenvolver um software didático sobre o cálculo de frenagem. O intuito é que ele sirva como objeto de aprendizagem em disciplinas de física do Ensino Médio, bem como em disciplinas de cursos superiores que abordem o tema. Havia a intenção de ser apresentado esse software em uma escola do Ensino Médio. No entanto, não foi possível executar esse teste, sendo apenas testado por alguns engenheiros. Contudo, os objetivos propostos foram alcançados.

Ao longo do processo, muitas dificuldades foram encontradas, principalmente na obtenção das fórmulas e dados das montadoras para que fosse possível obter os resultados.

Há vários trabalhos a serem desenvolvidos futuramente. Um deles é desenvolver o programa em outras linguagens de programação, a fim de possibilitar a incorporação de alguns recursos de usabilidade, como menus e botões. Para tanto, sugere-se Java ou SciLab. Outra possiblidade é disponibilizar o software para turmas do Ensino Médio e verificar se houve melhor entendimento do conteúdo a partir da introdução desse objeto de aprendizagem. Ademais, buscando uma maior precisão dos cálculos, seria possível aprofundar o tema e utilizar-se de equações que proporcionam, com mais precisão, a realidade do processo de frenagem.

\section{Referências}

Bornhold, A. M.. Dimensionamento de um sistema de freio para veículo offroad de pequeno porte. Monografia (Monografia do curso de Graduação em Engenharia Mecânica) Faculdade Horizontina, Horizontina, 2012.

Chevrolet. "Comparar versões: Chevrolet s10 cabine dupla”. Disponível: < https://www.chevrolet.com.br/byo$\mathrm{vc} / \mathrm{client} / \mathrm{pt} / \mathrm{BR} / \mathrm{chevrolet} / \mathrm{s} 10 / 2022 /$ double/features/trims/table? section=INFORMA\%C 3\%87\%C3\%95ES+COMPLEMENTARES\&styleOne=148TAN $>$ Acesso: Outubro/ 2021.

Fiat. "Fiat Strada Volcano". Disponível: < https://strada.fiat.com.br/\#/> Acesso: Outubro/ 2021.

Ford. "Ranger XLS 2.2 Diesel 4x4 AT 2022”. Disponível: < https://www.ford.com.br/picapes/ranger/compare-as-versoes/xls-22-diesel-4x4at/?intcmp=vhp-return-model $>$. Acesso: Outubro/2021.

Honda. "City LX 2021”. Disponível: < https://www.honda.com.br/automoveis/city\#exl> Acesso: Outubro/2021.

Hyundai. "HB20 2021 Nova geração". Disponível: < https://www.hyundai.com.br/linha-

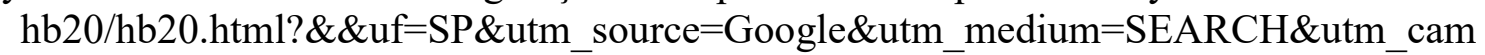
paign $=$ ONGOING_RETAIL_HB20_2021\&utm_content $=$ TEXT_HB20_VISION_GEN E_SC_HBNOVO_HE $>$ Acesso: Outubro/2021.

ISO 3450. (2011) "Earth-moving machinery - Wheeled or high-speed rubbertracked machines - Performance requirements and test procedures for brake systems". Disponível: 
http://106.38.59.21:8080/userfiles/b1cb859b78864bad9289f8ef5c446dfa/files/teckSoluti on/2019/07/EN\%20ISO\%203450\%EF\%BC\%9A2011.pdf> Acesso: Outubro/ 2021.

Maluf, O.; Angeloni, M.; Gualberto, A. R.; Spinelli, D.; Bose Filho, W. W. (2007) "Discos de freio automotivos: aspectos históricos e tecnológicos". Disponível: $<$ https://repositorio.pgsskroton.com/bitstream/123456789/1301/1/Artigo\%2020.pdf> Acesso: Outubro/2021.

Newton, I. Principia: princípios matemáticos de filosofia natural. São Paulo: Nova Stella / EDUSP, 2002.

Rache, M.. Mecânica Diesel: Caminhões - Pick-ups -Barcos. São Paulo: Editora Hemus, 2004.

Rodrigues, T. Física: Tempo de reação e de frenagem. São Paulo: Folha de São Paulo, 2002. Disponível: $\quad<$ http://www1.folha.uol.com.br/folha/educacao/ult305u11233.shtml> Acesso: Outubro / 2021.

Rosa, E.; Leal, L.C. M.; Nicolazzi, L.C. "Uma introdução à modelagem quase estática de veículos automotores de rodas". Publicação interna do GRANTE - Departamento de Engenharia Mecânica da Universidade Federal de Santa Catarina, Florianópolis, 2008. Disponível: < http://www. grante.ufsc.br>. Acesso: Julho / 2021.

Sales, G. L.; Maia, M. C.. Física Básica I. Fortaleza: UAB/IFCE, 2011.

Santos, G.C. M.. Projeto e dimensionamento de um sistema de freios aplicado e um veículo formula SAE. Monografia (Graduação em Engenharia Mecânica) - Escola Politécnica, Universidade Federal do Rio de Janeiro, Rio de Janeiro, 2014.

Silva, M. N. P.. "Calculando a Frenagem de um veículo". Disponível: $<$ http://mundoeducacao.bol.uol.com.br/matematica/calculando-frenagemum-automovel.htm>. Acesso: Julho / 2021.

Toyota. Comparar versões: Corolla d Disponível: < https://www.toyota.com.br/modelos/corolla/comparativo/ > Acesso: Outubro / 2021.

SIMRET 4000. Turnkey Instruments Ltd, junho 2021. Disponível: < https://turnkeyinstruments.com/product/simret-4000/>. Acesso: Setembro / 2021. 